\title{
Insights on the mechanical behavior of keratin fibrils
}

\author{
Egipto Antunes, Célia F. Cruz, Nuno G. Azoia, Artur Cavaco-Paulo* \\ CEB - Centre of Biological Engineering, University of Minho, 4710-057 Braga, Portugal
}

\section{A R T I C L E I N F O}

\section{Article history:}

Received 19 February 2016

Received in revised form 5 April 2016

Accepted 4 May 2016

Available online 6 May 2016

\section{Keywords:}

Molecular dynamics

Hair

Keratin

Pulling

Young's Modulus

\begin{abstract}
A B S T R A C T
A computational molecular model of a truncated keratin protofibril ( 8 chains of hair keratin, PDB provided in Supplementary material) was used, to run a series of steered molecular dynamics simulations obtaining strain-stress curves. These results were compared with experimental mechanical data on hair fibers. Our data demonstrate that the molecular dynamics simulations can model hair mechanical properties. Simulations done in vacuum showed a better agreement with experimental Young's Modulus(YM) values. The role of hydrogen bonds and the secondary structure of keratin on the mechanical properties was evaluated in detail. The incubation with a fragment of one surfactant protein, the SPD-2 peptide(QAAFSQ), showed the improvement of YM of the hair keratin either by simulations and experimental data. For the first, our research provides mechanistic insights on mechanical microscopic properties of keratin protofibrils through molecular dynamics simulations.
\end{abstract}

(c) 2016 Elsevier B.V. All rights reserved.

\section{Introduction}

Human hair is mainly composed by proteins and lipids (about $90 \%$ of proteins and $1-9 \%$ of lipids by dry weight), and can be divided into three zones: the cuticle (outermost zone), the cortex (middle zone) and in coarser hairs the medulla (innermost zone). The cuticle corresponds to a $5 \mu \mathrm{m}$ protective surface composed of several layers of overlapping scales. The medulla is made of hollow cells with amorphous material and air spaces, it has $5-10 \mu \mathrm{m}$ thickness and is not always present. The cortex, with $45-90 \mu \mathrm{m}$ of thickness, is responsible for $90 \%$ of hair mass. It is composed by long cylindrical assembled cortical cells and the cell membrane complex. These cells are constituted by macrofibrils and intermacrofibrilar material. The macrofibrils are further divided into intermediate filaments (IFS also known as microfibrils) and a matrix of associate proteins and remains of keratinocytes. The lateral assembling of coiled-coil $\alpha$-keratin ( $\alpha$-KRT) proteins and keratin associate proteins (KAPs) results in KRT dimers, tetramers, protofibrils (two tetramers) and finally IFS (4 protofibrils) [1,2]. The Fig. 1(a) represents this hierarchical structure of hair.

The hair and other keratinaceous fibers such as wools, quills and spines are widely studied at macroscopic level, from the response to chemical treatments [3-5] to inherent mechanical properties [6-9]. However at molecular level there is much lesser information about these fibers and their ultrastructure. This is probably due to

\footnotetext{
* Corresponding author.

E-mail address: artur@deb.uminho.pt (A. Cavaco-Paulo).
}

the limitations in the characterization of the entire IFS structure, since it has some highly disordered regions difficult to crystallize, challenging the use of traditional techniques such as X-ray crystallography and NMR. In a few works, computational methods have been applied with success in the study of these fibers structure at molecular level, overcoming these limitations. Computational molecular models of keratin structures (mainly dimers and some tetrameters of $\alpha$-keratin) have been developed, gathering the available data from several sources such as the known amino acid sequence of keratin proteins, the crystallographic structure of some keratin fragments already unveiled and available information about other fibrous proteins like vimentin and lamin which share similar ultrastructure of the IFS with keratin [10-13].

Previously a coarse-grained computational model of a truncated keratin protofibril (from the linker 2 region to the tail) was developed in our group, and it was used in several molecular dynamics simulations to study the interaction of hair protein content with alcoholic formulations. The performed simulation and experimental tests showed that the increase of a peptide affinity towards hair fibers can be modulated by the solvent composition of formulations [14]. In this work we intend to apply the molecular dynamics simulations in the study of mechanical properties of the truncated protofibril model and unveil how these properties are affected by the interaction with a fragment of one pulmonary protein, the SPD-2 peptide. We performed steered simulations, applying a pulling force to stretch the fiber and subsequently calculate the Young's Modulus (YM) in different conditions. For the same purpose, mechanical experimental tests with bleached 

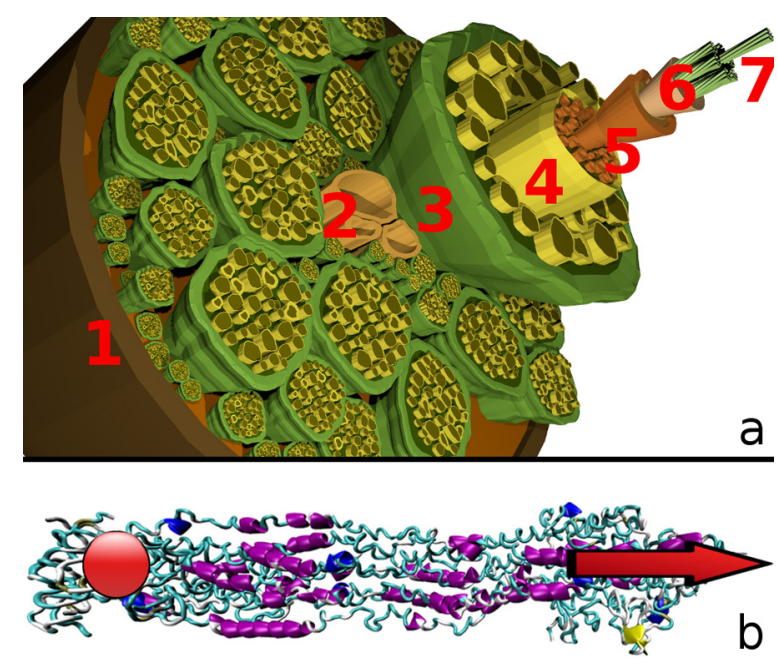

Fig. 1. (a) Schematic 3D representation of one hair shaft cut. 1 - Hair cuticle; 2 hollow medulla cells; 3 - cell membrane complex; 4 - macrofibril; 5 - matrix; 6 microfibrils or intermediate filaments; 7 - protofibril. (b) Schematic representation of steered simulations, the red ball indicates the fixed zone of the truncated protofibril and the arrow the zone where the pulling force is applied as well its direction. (For interpretation of the references to colour in this figure legend, the reader is referred to the web version of this article.)

Caucasian hair shafts were performed in similar conditions to the ones simulated, including the interaction with the peptide.

\section{Materials and methods}

\subsection{Molecular dynamics simulations}

A coarse-grained model of a truncated protofibril, assembling 8 truncated $\alpha$-keratins, was previously built by us [14]. The truncated zone corresponds to $2 \mathrm{~B}$ domain of the $\alpha$-keratins (from the L2 link to the $C$ tail). The model is composed by four keratin 34-keratin 86 parallel dimers, and each dimer assembles with the adjacent dimers in anti-parallel way according the $A_{22}$ model [14].

Although in this previous work the suitability of the coarsegrained model developed for the type of studies performed was shown [14], the MARTINI force-field [15] applied there do not allow dynamic conformational flexibility of the protein secondary structure due to its coarse-grained nature. This limits the study of mechanical studies proposed in the present work and in consequence the coarse-grained model was converted into a united-atom model by the "Backward" tool from the MARTINI force-field web site (http://md.chem.rug.nl/cgmartini/). The resulting united-atom model can be accessed in the Supplementary material as a PDB file.

All molecular dynamics simulations were performed with the Gromacs 4.0.7 package using the GROMOS96 43A1 united-atom force-field [16-18], with periodic boundary conditions, applying the LINCS [19] and the SETTLE [20] algorithms to constrain the peptide and water bonds respectively. The simulations ran with a time step of integration of $2 \mathrm{fs}$ and the single point charge (SPC) model [21] was used to represent the waters. When needed the temperature was maintained at $300 \mathrm{~K}$ with the V-rescale thermostat $[22,23]$ whereas the Parrinello-Rahman barostat $[24,25]$ (with semi-isotropic coupling) was used to maintain the pressure at $1 \mathrm{~atm}$. Van der Walls and electrostatic interactions were treated by a twin-range method, with short and long range cutoffs ( 0.8 and $1.4 \mathrm{~nm}$, respectively), with a reaction field correction for electrostatic using a dielectric constant of 54 [26], and the neighbor list was updated every 5 steps.

\subsubsection{Preparation simulations}

Initially all systems were energy minimized for around 2000 steps with the steepest descent method. Afterwards the systems were initiated to a series of three short equilibration simulations (500 ps each simulation), with positions restraints allowing the slow relaxation of the structures for the final production run (the atoms were restrained with an harmonic force of $10^{3} \mathrm{~kJ} / \mathrm{mol} \mathrm{nm}^{2}$ ). In the aqueous systems the equilibration simulations consisted of three short runs. The first in the NVT ensemble (number of particles, volume and temperature constant) applying positions restraints to all heavy atoms. The second one simulation in the NPT ensemble (number of particles, pressure and temperature constant) with positions restraints to the same atoms. Finally the third simulation ran also in NPT ensemble but with positions restrains only in the alpha-carbons atoms of the proteins. The equilibration simulations of the vacuum systems followed the same protocol in terms of positions restrains, but in all the three simulations, the NVE ensemble (number of particles, volume and energy constant) was applied.

After the systems equilibration by the preparatory simulations, were performed $50 \mathrm{~ns}$ of NPT (aqueous systems) or NVE (vacuum systems) simulations, without any position restrains. This allowed the keratin chains to acquire a stable conformation in response to their surrounding (water or vacuum) as well to the interaction with the SPD-2 peptide when presented.

\subsubsection{SPD-2 peptide}

The SPD-2 peptide (QAAFSQ) is a fragment of pulmonary associated surfactant protein $\mathrm{D}$ (SPD), that belongs to a group of proteins present in the lung which have been studied in our group as enhancer agents in the recovery of damaged hair [14,27-29]. The computational model of the peptide was built using the Pymol software [30]. The peptide model was subject to preparation simulations by the same protocol described previously. After the equilibration, the resulting systems were subject to 50 ns of simulations without any position restraints to relax the peptide structure. The resulting structures were used to test the effect of this peptide on the keratin fiber, inserting 10 molecules in the simulation box and following the previous protocol for systems equilibration.

\subsubsection{Steered simulations}

The final conformations of the previous runs were used to initiate the steered molecular dynamics simulations, where a spring elastic constant force was applied to one tip of the keratin fiber whilst the other tip was fixed, promoting the protofibril stretch and allowing the study of mechanical properties such as the YM. The centers of mass of the first amino acids, in the region $2 \mathrm{~B}$ of each keratin fiber, were fixed with a strong harmonic restraint with a spring constant of $10^{3} \mathrm{~kJ} / \mathrm{mol} \mathrm{nm}^{2}$, while the last amino acids at the opposite end of the same region were pulled by a spring with an elastic constant of $100,000 \mathrm{~kJ} \mathrm{~mol}^{-1} \mathrm{~nm}^{-2}$ (see Fig. $1 \mathrm{~b}$ ). The aqueous systems were simulated in an NPT ensemble while the vacuum system ran in an NVE ensemble. The pulling force (F) and the distance between the fixed and the pulled groups (L) were recorded during the simulation allowing the calculation of the engineered stress $(\sigma)$ and strain $(\varepsilon)$, which are described as:

$\sigma=\frac{F}{A_{0}}$

and

$\varepsilon=\frac{\Delta L}{L_{0}}$

where $\mathrm{F}, \mathrm{A} 0, \Delta \mathrm{L}$ and $\mathrm{L} 0$ are the pulling force, the cross-sectional area of the fiber, the displacement and the initial length, respectively. YM is a mechanical property that relates the engineered 
stress and strain providing a measure of the material stiffness. It can be calculated using the following expression:

$Y M=\frac{\sigma}{\varepsilon}=\frac{\frac{F}{A_{0}}}{\frac{\Delta L}{L_{0}}}$

\subsection{Hair treatments}

Caucasian hair fiber samples were provided by International Hair Importers \& Products (New York). Hair tresses were subjected to eight cycles of bleaching, which destroys the hair exterior lipid layer and damages hair cuticle, reducing its influence in the mechanical tests and contributing to a keratin fiber more similar to the one simulated. Each bleaching cycle consisted of the application of $10 \% \mathrm{H}_{2} \mathrm{O}_{2}(\mathrm{v} / \mathrm{v})$ in $\mathrm{Na}_{2} \mathrm{CO}_{3} / \mathrm{NaHCO} \mathrm{pH} 9.0$ buffer at $50^{\circ} \mathrm{C}$ for $1 \mathrm{~h}$. Between cycles, the hair was washed with running water.

Subsequently, the bleached hair was treated with $700 \mu \mathrm{M}$ of the SPD-2 peptide in $0.05 \mathrm{M} \mathrm{PB}, \mathrm{pH}$ 7.5. The engineered peptide was synthesized by JPT Peptide Technologies GmbH (Berlin, Germany). Peptide treatments were performed for $2 \mathrm{~h}$ at $37^{\circ} \mathrm{C}$ with orbital agitation, $50 \mathrm{rpm}$. After treatment, the hair samples were washed with distilled water.

\subsection{Mechanical properties evaluation}

Hair samples mechanical properties were evaluated following the guidelines for tensile testing of fibers outlined in ASTM D1445-95. Measurements were performed in an Instron 4505 tensile tester, with a maximum load cell capacity of $2.5 \mathrm{~N}$. A set of 15 single hair fibers was randomly taken from the hair tresses for each sample measurement. Each hair fiber was individually mounted in the tensile jig through a paper scaffold with a fixed gauge length of $20 \mathrm{~mm}$. Before the test started, the scaffold was cut across, so the hair fiber was fixed in a continuous length within the jig. All the samples were kept under the same conditions before measurements. The wet samples were soaked in distilled water for at least $5 \mathrm{~min}$ previous to the measurement. Measurements were performed at a constant rate of $1.5 \mathrm{~mm} / \mathrm{min}$ until breakage, under $60 \pm 5 \%$ relative humidity. For each hair, the applied load against extension was recorded, assuming an average mean diameter of $70 \mu \mathrm{m}$ (measured in previous studies by light microscopy on transversal cut hair samples). All data were converted to stress (load/unit area) against strain (\% extension).

\section{Results and discussion}

Fig. 2 shows the time evolution of protofibril in water and in vacuum steered simulations. The stretching of the chains and the consequent structural deformation are clear. The first zones to deform in response to the applied force are the less coiled zones. With the time evolution the more coiled domains, like the $\alpha$-helix (at violet in Fig. 2), also begin to stretch and unfold (this behavior can be better perceived in the video 1 of Supplementary material). Although the initial conformation of the truncated protofibril in vacuum is more compact, the fibers are less structured, a fact that in conjugation with the absence of water, seems facilitate the fiber stretching.

The DSSP algorithm ("Define Secondary Structure of Proteins") [31] describes the amino acids secondary structure and provides a more reliable information about the protofibril conformation. The variation of the secondary motifs $\alpha$-helix and $\beta$-sheets along the simulated time for all the systems is represented in Fig. 3.

In the aqueous simulations there is a clear decrease in number of amino acids in the form of $\alpha$-helix, which is expected in response to the fiber stretching. The vacuum systems almost do not present

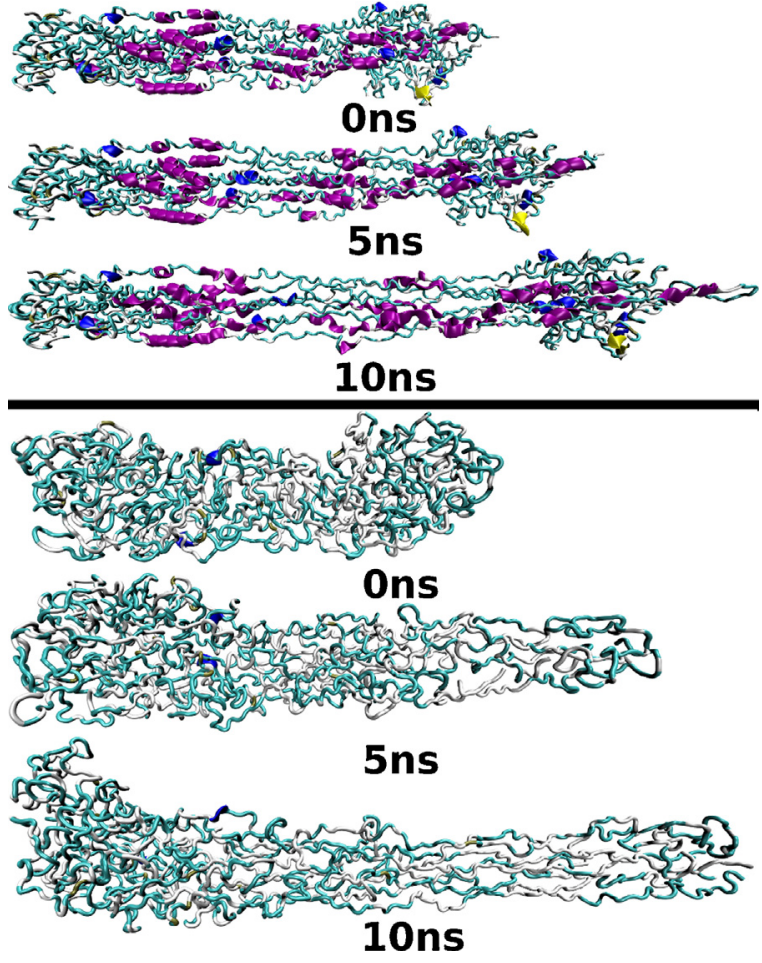

Fig. 2. Snapshots of aqueous (up) and vacuum (down) steered simulations. The centers of mass of the first amino acids at left in the region $2 \mathrm{~B}$ of each keratin fiber were fixed while the first right amino acids of the same region were pulled.

$\alpha$-helix motifs and their number is almost constant during the simulations, showing a tiny initial increase that could be a result of the unfold of the fibers from an initial highly compact conformation. On the contrary, the $\beta$-sheets motifs slightly increase along the simulation except for the fiber in water system. There is an initial $\beta$-sheets decrease in the vacuum systems, again probably due to the initially high compaction of the structure, and also a decrease at $4 \mathrm{~ns}$ of simulation of the protofibril in water. The $\alpha$-helix to $\beta$-sheet transition in response to the keratin fiber stretching is described in literature for more than 50 years [32,33], and although very small, some $\alpha$ helix to $\beta$-sheet transition is shown in the previous results and in the simulations pictures.

In Fig. 4a the averaged strain-stress curves resulting from the pulling simulations are presented. Several works, either theoretical or experimental, with keratin filaments such as hair and wool, or even with other intermediate filaments like vimentin describe three regions in its strain-stress graphics [34-37], namely:

I-“Linear elastic region" or "Hookean region" (0-2\% of strain): characterized by the linear increase of the stress with the strain until a plateau is reached;

II-"Yield region" (2-30\% of strain): the stress maintains almost constantly over a long period;

III- "Post yield region" (above 30\% of strain): the stress increases rapidly with the strain until the fiber failure.

The identification of these three zones in the strain-stress curves is not always easy, or even possible, due to big variations of the slope [11,36].

As shown in Fig. 4a, the identification of the three zones in our simulations was only possible for the truncated protofibril in water (curve W). For these systems the Hookean region seems to end around 0.1 of strain (10\% of fiber elongation) and the Yield region around 0.35 . Although the stress values are not constant between the strain values of 0.1 and 0.35 its increase is much lower than in the other regions of the curve. For the remaining systems the division of the graphs in three zones is not clear. The strain-stress graph 

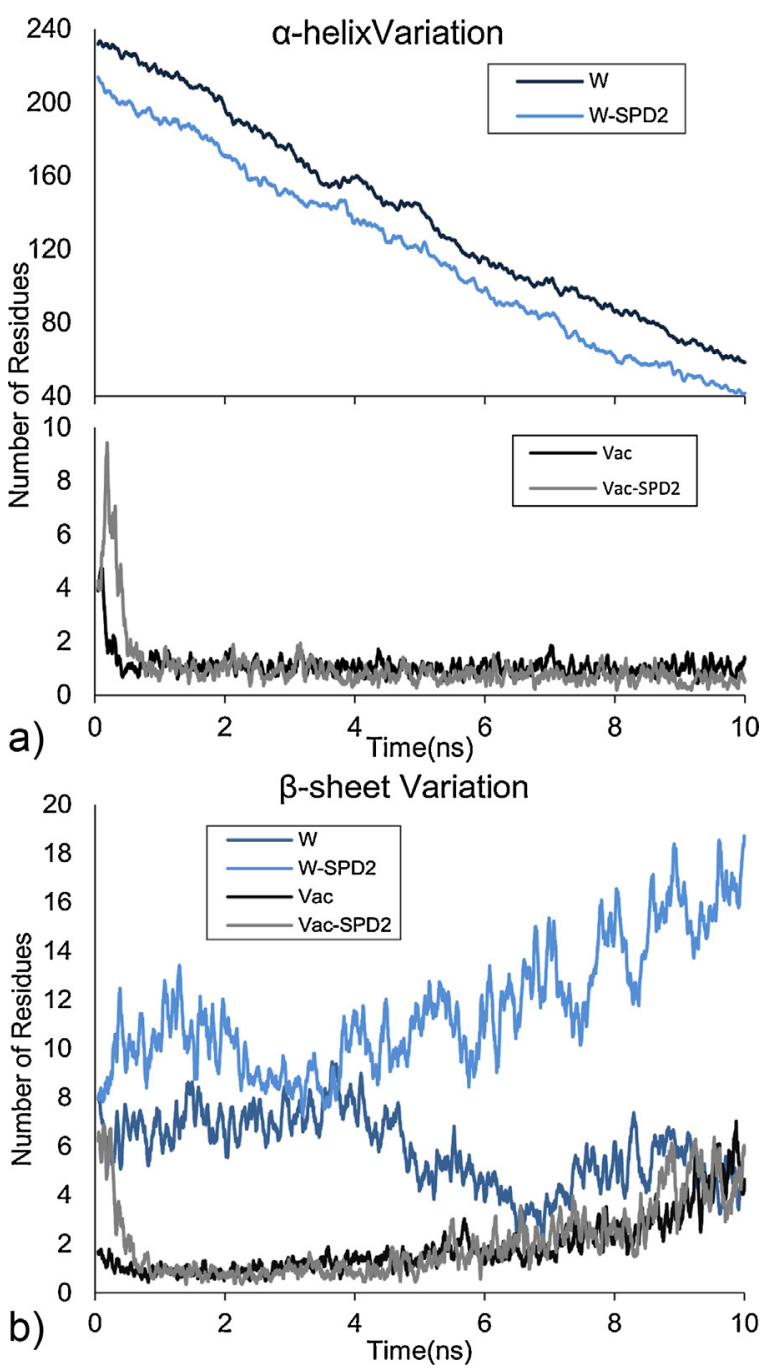

Fig. 3. Graphical representation of the number of residues in $\alpha$-helix (a) and $\beta$-sheet (b) conformation along the simulation time. The variation of $\alpha$-helix residues from aqueous and vacuum simulations is separated due the difference in values range. W: truncated protofibril in water; Vac: truncated protofibril in vacuum simulations.

for the protofibril in vacuum (curve Vac in Fig. 4a) is clearly different of the aqueous system, showing an almost constant increase of the stress values along the simulation. The aqueous systems with the peptide (W-SPD2 in Fig. 4a) seems to have only the regions I and II. Just as in the simulations of the fiber alone in water there is a quick increase of stress values until around 0.1 of fiber strain, but after this point the stress increases with almost constant rate until the end of the simulations. On the other hand the vacuum systems with SPD-2 (Vac-SPD2 in Fig. 4a) presented a constant increase of the stress values until 0.35 of strain, where the slope of the curve increases, opposing to the behavior of the fiber in vacuum. In this case the slope change is an indication of the transition from the region II to III.

Fig. $4 \mathrm{~b}$ presents the same study for the real hair shafts. Taking into consideration the typical strain-stress curve for keratin and the global behavior of all 15 replicas per condition (in Supplementary material all the curves are presented), only one replica is represented. On the contrary to the simulation results, the stress values of dry hair are higher to ones for wet hair. However, like in the theoretical tests, the maximum strain values for dry hair samples are higher. The addition of the peptide clearly affects the strain stress curves in both conditions, resulting in the increase of both the stress and strain values. This increase in stress promoted by the

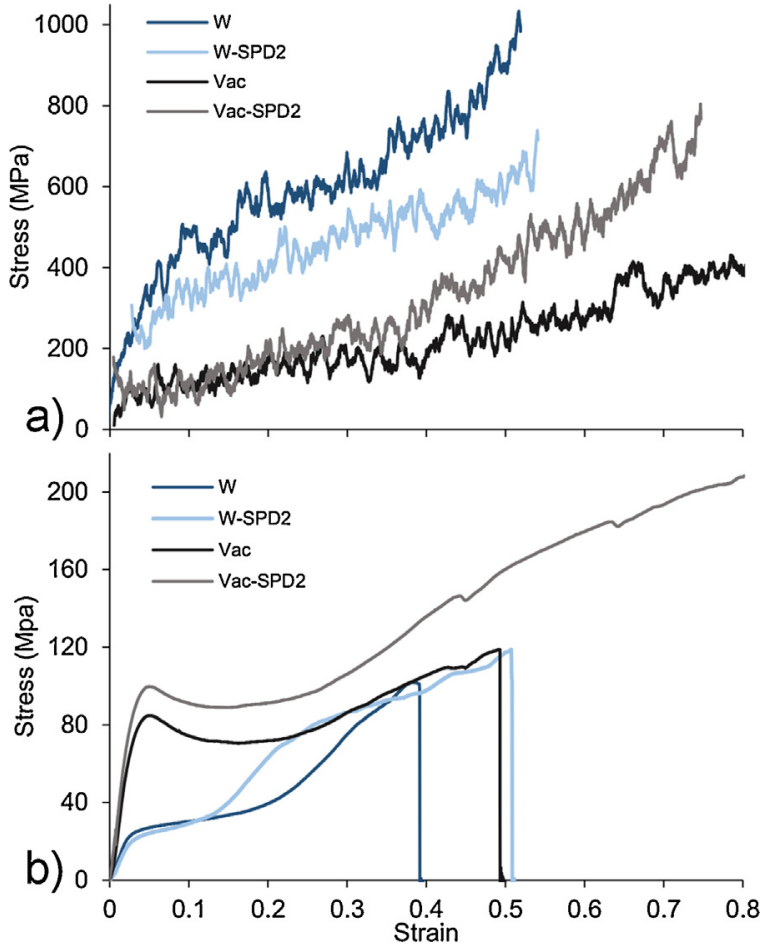

Fig. 4. (a) Mechanical analysis of the truncated protofibril under steered simulations. (b) Mechanical analysis of hair shafts under tensile deformation. W: truncated protofibril in water simulations or humid hair shafts; Vac: truncated protofibril in vacuum simulations or dry hair shafts.

SPD-2 treatment begin much earlier in dry hair samples ( $1 \%$ in dry hair elongation against $10 \%$ for wet samples). In these strain-stress curves it is easy to define the elastic zone. It ends around 0.02 and 0.05 of strain for wet and dry samples, respectively. The transition from the yield to the post yield region is harder to define, mainly for the dry samples results. It seems to happen at $10 \%$ of elongation for the wet hair samples, incubated with peptides and at around $20 \%$ for the remaining samples.

Fig. 5 shows the Young's Modulus (YM) for the different conditions. The variation in these values shows a good agreement between simulation and mechanical assays. Nevertheless the calculated YM value for the simulations of the protofibril in water is higher than it is supposed to be, being the highest value calculated for all simulations. This value was expected to be lower than the value calculated for the protofibril in vacuum. This variation also contradicts the literature since it is well known that water molecules are capable of interacting with hair fibers plasticizing

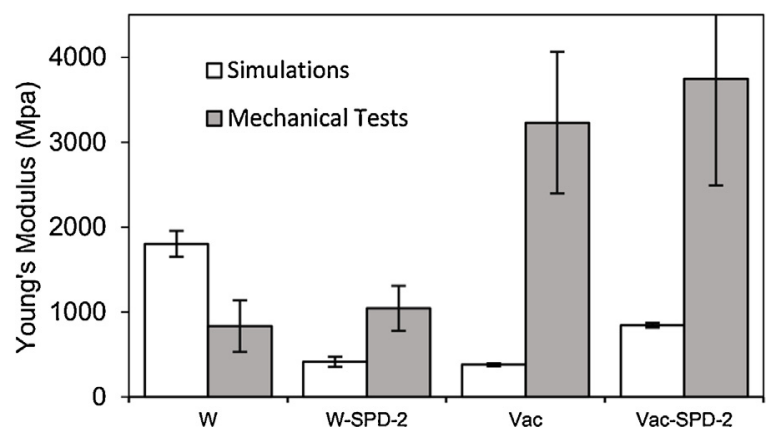

Fig. 5. Young's Modulus values calculated from simulations (white columns) and mechanical tests (gray columns) W: truncated protofibril in water simulations or humid hair shafts; Vac: truncated protofibril in vacuum simulations or dry hair shafts. 

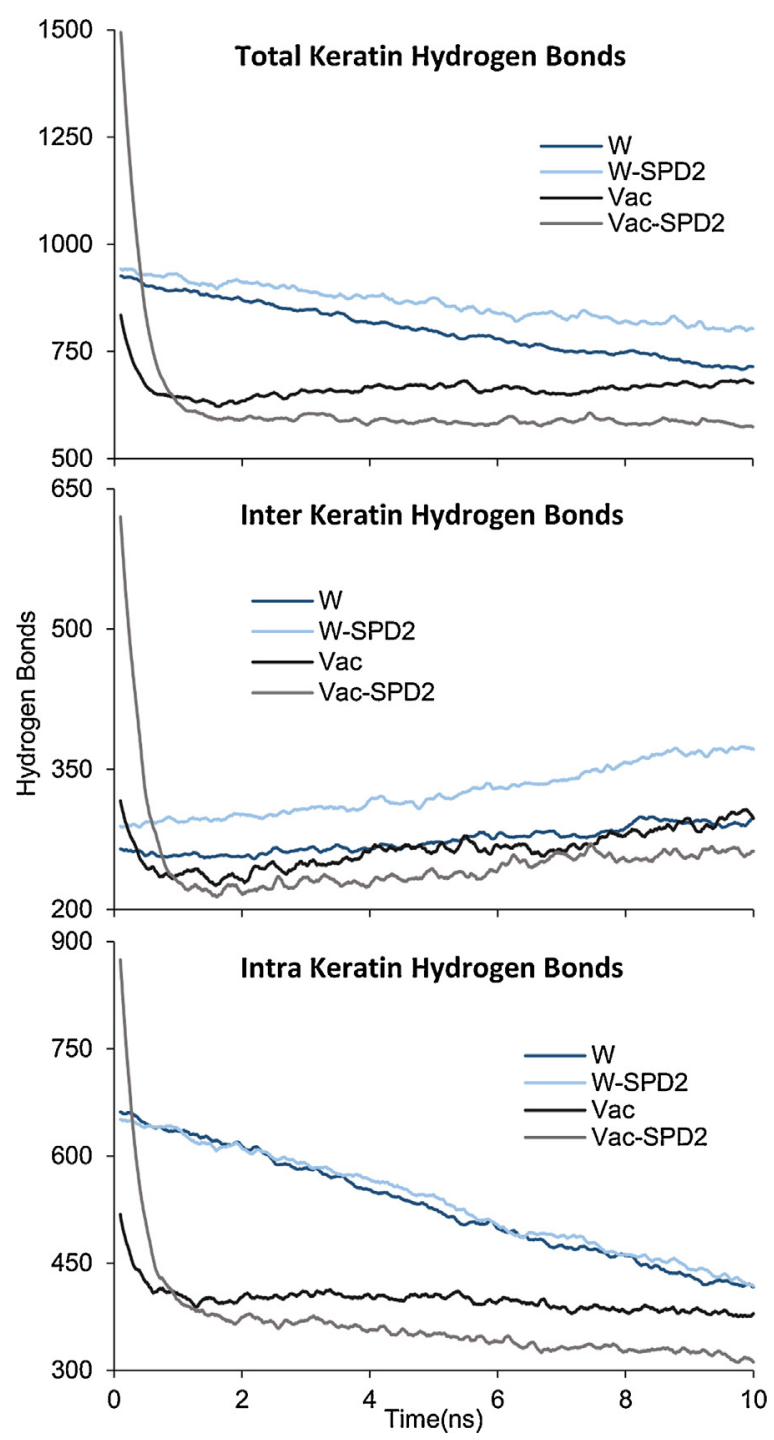

Fig. 6. Hydrogen bonds between keratin residues along the simulation. Up: total hydrogen bonds variation (total HB); middle: variation of hydrogen bonds between residues of the same keratin chain (intra-HB); down: variation of hydrogen bonds between residues of the different keratin chains (inter-HB). W: truncated protofibril in water simulations; Vac: truncated protofibril in vacuum simulations.

their components, and resulting in a lower force requirement for extending the fibers and consequently lower the elastic modulus $[6,35]$. However, this water effect is observed for the simulations with SPD-2, where the YM values from the aqueous simulations are lower than the corresponding values for the vacuum simulations.

Hydrogen bonds (HB) variation was studied in an attempt to unveil the mechanisms behind the previous results. The HB between atoms from different (inter-HB) and from the same keratin chains (intra-HB) were evaluated. The averaged results are presented in Fig. 6. The total HB number between protofibril atoms (inter and intra-HB) decreases along the simulation for all aqueous systems, being more pronounced when the protofibril is not accompanied by the peptide. This decrease is expected due to the stretching of the keratin chains that leads to the break of the initial $\mathrm{HB}$. However the vacuum systems only presented significant HB decrease at the beginning of the simulation, and in the protofibril in vacuum system (Vac in Fig. 6) the total HB begin to increase after 2 ns of simulation. The separate study of inter and intra-HB (Fig. 6) reveals additional interesting information.
The first noticeable feature is that the HB between keratin residues of the same chain (intra-HB) are in a higher number than the HB between residues from different chains (inter HB). There is a small increase of inter-HB during the simulation for all systems, opposing to the intra- $\mathrm{HB}$, that are almost constant in the protofibril in vacuum systems and decrease in the remaining systems. The inter and intra-HB graphs also depicted that the difference between the final number of total protofibril HB in water systems is due to the inter- $\mathrm{HB}$, since the peptide presence leads to a bigger increase of inter-HB in these systems, whereas the intra-HB are very similar in the two aqueous systems. The competition for HB donors and acceptors can explain this phenomena, namely the presence of the peptides that can limit the access of some keratin atoms to water molecules that otherwise would make HB with these waters. Once free, these keratin atoms can connect with other atoms from the surrounding chains, increasing the inter-HB. This also agrees with the fact that the peptide presence does not significantly affect the number of inter-HB in vacuum systems. Note that some of the inter$\mathrm{HB}$ increase is simply due to the keratin chain stretching, since there is an increase of these $\mathrm{HB}$ in all the conditions.

The difference of final number, in vacuum systems, of total HB is mainly due to the intra-HB that decrease less when peptides are not presented. In this case the competition could be between acceptors and donors from the same chain versus from the chain and peptides; there is less available spots to make HB inside the same chain because some of them are making $\mathrm{HB}$ with the adjacent peptides.

The observed overall decrease of $\mathrm{HB}$ is expected, the fiber stretching leads to the breakage of the HB. The vacuum systems presented one faster decrease of protofibril HB, probably as a result of the initial highest compaction of the fibers due to the vacuum, which allows more HB connections. This compaction is not natural and the fiber quickly expands with the applied stress force, breaking many of these bonds.

All together the results showed that the molecular dynamics simulations can be a useful and valuable research tool in the study of mechanical properties of hair fibers, although precaution is needed in the simulation results analysis. The strain-stress curves of the mechanical tests showed a typical profile for keratinaceous materials, while in the simulations only the systems with protofibril in water presented more clearly the three discussed regions. In addition, the stress values of vacuum systems in the simulations results are lower than ones for the aqueous simulations, which goes against the literature and our mechanical results. Other theoretical studies with hair keratin structures, presented strain-stress curves with the three elastic, yield and post yield regions clearly identified, but in some cases the curves presented are also different from the typical $[11,36]$. Chou and Buehler performed pulling simulations in three hair keratin structures [11], namely in one dimer, one truncated tetramer without cysteine bonds and one truncated tetramer with cysteine bonds (between the adjacent dimers). All the three conditions presented different strain-stress curves and only one from the dimer structure is similar to the typical. This shows that in these simpler keratinaceous systems, comparing to the more complex structures present in the hair, small changes can affect the strain-stress response. The strain-stress graphs of keratin dimers simulations with cysteine bonds between its two chains in Duchstein et al. work [38] did not present significant differences for the same study in the Chou and Buehler work, which seems to indicate that the absence of cysteine bonds in our model is not the cause of these inconsistencies.

The YM results presented a good agreement between simulation and mechanical results, except for the simulations of the protofibril in water. Previous studies in our research group, depicted big affinity of the surfactant pulmonary peptides with hair keratin and its potential as helper agents in the recovering of damaged hair mechanical properties $[14,27,28]$. In consequence we expected an 
increase of YM values for the aqueous systems when the peptides are present. These previous works findings, the mechanical tests results and the bigger stress values for aqueous systems seem to point to an overestimation of stress and YM values for aqueous simulations that is larger when the peptide is absent. This overestimation can be result of an increase of protofibril rigidity due to the protofibril interaction with the bulk water in the aqueous simulations, which could be minimized by the peptide presence. The water molecules surrounding the keratin fiber seem to promote more stable keratin-keratin HB, mainly the intra-HB. It should be remembered that the keratin fibers in the hair are surrounded by proteinaceous materials and although the water content in the hair interior varies with the environmental humidity, the keratinous fibers are not surrounded by bulk water. The YM results for the vacuum simulation and the dry hair shafts, on the contrary, presented similar variation, although the simulations values are around 5-8 times smaller. The presence of the peptide in the vacuum simulations increases the YM of the protofibril as expected from the previous works. These results show that the vacuum simulations can be used to qualitatively evaluate peptides effects on the mechanical properties of hair keratin structures.

The SPD-2 peptide leads to the increase of hair resistance to deformation, since its addition increases the stress values in the mechanical tests, as well as the YM in the mechanical tests and vacuum simulations. Thus, the potential of surfactant pulmonary peptides as helper agents in the recovery of damaged hair is confirmed.

\section{Conclusions}

In the last years many researchers have driven efforts to unveil the molecular structure properties of keratin fibers, trying to relate them with the macroscopic properties of hair tresses.

In this work we showed that molecular dynamics simulations can have an active role in the connection between the molecular and macroscopic study of hair. The application of steered molecular dynamics simulations, in a previously developed computational model of hair truncated protofibril, provided information about its mechanical properties compatible with laboratory tests performed with hair shafts. Vacuum simulations and laboratory mechanical tests showed an increase of YM after SPD-2 treatment, confirming its capacity to improve mechanical properties of hair, already observed in previous works. The agreement between these simulation results with the laboratory tests, as well as with previous findings about the surfactant pulmonary peptides effects on hair, demonstrates the potential of molecular dynamics simulations and also of the used model, in the study of mechanical properties of hair keratin fibers and the way these properties are affected by peptides treatment.

\section{Acknowledgments}

The authors acknowledge the access of Minho University "SeARCH" ("Services and Advanced Research Computing with HTC/HPC clusters") cluster. We also thank the Portuguese Foundation for Science and Technology (FCT) for providing Célia F. Cruz the grant for PhD studies (scholarship SFRH/BD/100927/2014).

\section{Appendix A. Supplementary data}

Supplementary data associated with this article can be found, in the online version, at http://dx.doi.org/10.1016/j.ijbiomac.2016. 05.018.

\section{References}

[1] L.J. Wolfram, Human hair: a unique physicochemical composite, J. Am. Acad. Dermatol. 48 (2003) S106-14.

[2] C. Popescu, H. Höcker, Hair-the most sophisticated biological composite material, Chem. Soc. Rev. 36 (2007) 1282-1291.

[3] R. Dawber, Hair: its structure and response to cosmetic preparations, Clin. Dermatol. 14 (1996) 105-112.

[4] C. Weathersby, A. McMichael, Brazilian keratin hair treatment: a review, J. Cosmet. Dermatol. 12 (2013) 144-148.

[5] A. Pielesz, A. Wlochowicz, W. Biniaś, Evaluation of structural changes in wool fibre keratin treated with azo dyes by Fourier Transform Infrared Spectroscopy, Spectrochim. Acta A Mol. Biomol. Spectrosc. 56 (2000) 1409-1420.

[6] T. Gao, Evaluation of hair humidity resistance/moisturization from hair elasticity, J. Cosmet. Sci. 58 (2007) 393-404.

[7] H. Tohmyoh, M. Ishihara, M. a. Salam Akanda, S. Yamaki, T. Watanabe, T. Iwabuchi, Accurate determination of the structural elasticity of human hair by a small-scale bending test, J. Biomech. 44 (2011) 2833-2837.

[8] K. Jelen, M. Skřontová, L. Šimkova, J. Zeman, E. Tlapáková, O. Fanta, Changes in the mechanical parameters of hair in a group of women in reproductive age, Neuro Endocrinol. Lett. 35 (2014) 481-489.

[9] W. Yang, J. McKittrick, Separating the influence of the cortex and foam on the mechanical properties of porcupine quills, Acta Biomater. 9 (2013) 9065-9074.

[10] C.-H. Lee, M.-S. Kim, B.M. Chung, D.J. Leahy, P.A. Coulombe, Structural basis for heteromeric assembly and perinuclear organization of keratin filaments, Nat. Struct. Mol. Biol. 19 (2012) 707-715.

[11] C.-C. Chou, M.J. Buehler, Structure and mechanical properties of human trichocyte keratin intermediate filament protein, Biomacromolecules 13 (2012) 3522-3532

[12] C. Danciulescu, B. Nick, F.-J. Wortmann, Structural stability of wild type and mutated alpha-keratin fragments: molecular dynamics and free energy calculations, Biomacromolecules 5 (2004) 2165-2175.

[13] Z. Qin, M.J. Buehler, Computational and theoretical modeling of intermediate filament networks: structure, mechanics and disease, Acta Mech. Sin. Xuebao 2012 (2016) 941-950.

[14] E. Antunes, C.F. Cruz, N.G. Azoia, A. Cavaco-Paulo, The effects of solvent composition on the affinity of a peptide towards hair keratin: experimental and molecular dynamics data, RSC Adv. 5 (2015) 12365-12371.

[15] S.J. Marrink, H.J. Risselada, S. Yefimov, D.P. Tieleman, A.H. de Vries, The MARTINI force field: coarse grained model for biomolecular simulations, J. Phys. Chem. B. 111 (2007) 7812-7824.

[16] H.J.C. Berendsen, D. van der Spoel, R. van Drunen, GROMACS: a message-passing parallel molecular dynamics implementation, Comput. Phys. Commun. 91 (1995) 43-56.

[17] E. Lindahl, B. Hess, D. van der Spoel, GROMACS 3.0: a package for molecular simulation and trajectory analysis, J. Mol. Model. 7 (2001) 306-317.

[18] W.R.P. Scott, P.H. Hu, I.G. Tironi, A.E. Mark, S.R. Billeter, J. Fennen, et al., The GROMOS biomolecular simulation program package, J. Phys. Chem. (1999) 3596-3607.

[19] B. Hess, H. Bekker, H.J.C. Berendsen, J.G.E.M. Fraaije, LINCS: a linear constraint solver for molecular simulations, J. Comput. Chem. 18 (1997) 1463-1472.

[20] S. Miyamoto, P.A. Kollman, Settle: an analytical version of the SHAKE and RATTLE algorithm for rigid water models, J. Comput. Chem. 13 (1992) 952-962.

[21] J. Hermans, H.J.C. Berendsen, W.F. Van Gunsteren, J.P.M. Postma, A consistent empirical potential for water-protein interactions, Biopolymers 23 (1984) 1513-1518.

[22] H.J.C. Berendsen, J.P.M. Postma, W.F. van Gunsteren, A. DiNola, J.R. Haak, Molecular dynamics with coupling to an external bath, J. Chem. Phys. 81 (1984) 3684

[23] G. Bussi, D. Donadio, M. Parrinello, Canonical sampling through velocity rescaling, J. Chem. Phys. 126 (2007) 014101.

[24] M. Parrinello, A. Rahman, Polymorphic transitions in single crystals: a new molecular dynamics method, J. Appl. Phys. 52 (1981) 7182-7190.

[25] S. Nosé, M.L. Klein, Constant pressure molecular dynamics for molecular systems, Mol. Phys. 50 (1983) 1055-1076.

[26] P.E. Smith, W.F. van Gunsteren, Consistent dielectric properties of the simple point charge and extended simple point charge water models at 277 and 300 K, J. Chem. Phys. 100 (1994) 3169.

[27] N.G. Azoia, M.M. Fernandes, N.M. Micaêlo, C.M. Soares, A. Cavaco-Paulo, Molecular modeling of hair keratin/peptide complex: using MM-PBSA calculations to describe experimental binding results, Proteins Struct. Funct. Bioinform. 80 (2012) 1409-1417.

[28] M. Fernandes, A. Cavaco-Paulo, Protein disulphide isomerase-mediated grafting of cysteine-containing peptides onto over-bleached hair, Biocatal. Biotransform. 30 (2012) 10-19.

[29] M.M. Fernandes, A. Cavaco-Paulo, Protein disulphide isomerase-assisted functionalization of proteinaceous substrates, Biocatal. Biotransform. 30 (2012) 111-124.

[30] Schrodinger LLC, The PyMOL Molecular Graphics System, Version 1.1, (2010).

[31] W. Kabsch, C. Sander, Dictionary of protein secondary structure: pattern recognition of hydrogen-bonded and geometrical features, Biopolymers 22 (1983) 2577-2637 
[32] W.T. Astbury, H.J. Woods, X-ray studies of the structure of hair wool, and related fibres. II.-the molecular structure and elastic properties of hair keratin, Proc. R. Soc. B Biol. Sci. 114 (1934) 314-316.

[33] E.G. BENDIT, The $\alpha-\beta$ transformation in keratin, Nature 179 (1957) 535.

[34] B.M. Chapman, 15-A review of the mechanical properties of keratin fibres, J. Text. Inst. 60 (1969) 181-207.

[35] J. McKittrick, P.Y. Chen, S.G. Bodde, W. Yang, E.E. Novitskaya, M.A. Meyers, The structure, functions, and mechanical properties of keratin, JOM 64 (2012) 449-468.
[36] C.-C. Chou, E. Lepore, P. Antonaci, N. Pugno, M.J. Buehler, Mechanics of trichocyte alpha-keratin fibers: experiment, theory, and simulation, J. Mater. Res. 30 (2015) 26-35.

[37] Z. Qin, L. Kreplak, M.J. Buehler, Hierarchical structure controls nanomechanical properties of vimentin intermediate filaments, PLoS One 4 (2009) e7294.

[38] P. Duchstein, T. Clark, D. Zahn, Atomistic modeling of a KRT35/KRT85 keratin dimer: folding in aqueous solution and unfolding under tensile load, Phys. Chem. Chem. Phys. 17 (2015) 21880-21884. 